02

\title{
Определение констант скоростей и механизма фотопревращений металлопорфиринов методом решения обратной фотокинетической задачи
}

\author{
(C) И.В. Станишевский, С.М. Арабей \\ Белорусский государственный аграрный технический университет, \\ 220023 Минск, Республика Беларусь \\ e-mail: ivanstanisheuski@mail.ru
}

Поступила в редакцию 23.06.2020 г.

В окончательной редакции 28.08.2020 г.

Принята к публикации 24.09.2020 г.

\begin{abstract}
Методами обратной кинетической задачи исследованы кинетики флуоресценции некоторых замещенных $\mathrm{Zn}$ - и $\mathrm{Mg}$-порфиринов в твердых полимерных пленках при возбуждении двухступенчатыми прямоугольными лазерными импульсами и $293 \mathrm{~K}$. Экспериментальные немонотонные кинетические кривые аппроксимированы симулированными в рамках шестиуровневой энергетической схемы, описывающей обратимые взаимопревращения двух комплексов. Максимальное соответствие экспериментальных и симулированных кривых получено итерационной оптимизацией с использованием алгоритма Нелдера-Мида. На основании статистически оцененных значений констант скоростей и параметров рассмотренных моделей дана интерпретация наблюденных кинетик и сделан вывод о том, что взаимопревращения вызваны процессом обратимого экстралигандирования центрального иона металла, протекающим в возбужденном триплетном $T_{1}$-состоянии.

Ключевые слова: обратная кинетическая задача, обратная фотокинетическая задача, двухступенчатые прямоугольные импульсы фотовозбуждения, оценка фотокинетических параметров, фейдинг флуоресценции, антифейдинг флуоресценции, экстра-лигандирование, алгоритм Нелдера-Мида.
\end{abstract}

DOI: 10.21883/OS.2021.02.50552.180-20

\section{Введение}

Большинство исследований кинетики вторичного свечения в частности, органических люминесцирующих соединений при импульсном фотовозбуждении (ФВ), направлено на получение информации о скоростях распада и образования возбужденных электронных состояний, а также скоростях протекания фотофизических и фотохимических процессов. Проводимый нелинейный регрессионный анализ кривых по сути является начальным и единственным этапом последовательного формальнокинетического подхода, определенного как обратная кинетическая задача (ОКЗ) [1-3]. При этом полный алгоритм решения ОКЗ более сложный и гораздо более ресурсоемкий: на последующих этапах происходит оценка кинетических параметров или/и дискриминация гипотез [1]. В обоих случаях сначала задается математическая (гипотетическая) модель фотопроцесса в виде системы дифференциальных уравнений (СДУ). Затем численными итерационными методами решается задача минимизации(оптимизации) целевой функции (ЦФ) функции „рассогласования“ между экспериментальной и модельной кривой. Таким образом, формально ОКЗ представляет собой задачу нахождения таких значений кинетических и других расчетных параметров, при которых достигается наилучшее в статистическом смысле описание экспериментальных данных при физическом смысле гипотетической модели. Известно, что в силу ряда причин ОКЗ является некорректной, так как не имеет единственного решения [1-4], поскольку ЦФ может достигать минимума для множества наборов параметров модели. Часто дополнительной трудностью является длительное время оптимизации ЦФ из-за необходимости многократного интегрирования СДУ (во многих случаях „жесткого“ типа).

Следует отметить, что весьма незначительное число физико-химических исследований, посвященных оценке параметров модели, выполнено в рамках формальнокинетического подхода [5-8]. При этом в большинстве работ ОКЗ решались приближенными методами. Такое, сохраняющееся уже много лет состояние проблемы объясняется рядом обстоятельств: отсутствием необходимых экспериментальных методов и готовых к использованию программных алгоритмов, а также значительными требованиями к вычислительной мощности компьютеров при возрастании количества оцениваемых параметров.

В работах [9-12] предложен и реализован новый метод определения констант скоростей фотоиндуцированных реакций в органических и комплексных соединениях, основанный на формально-кинетическом подходе к решению обратных фотокинетических задач (ОФКЗ). Эти работы стали продолжением модельных компьютерных экспериментов, показавших, что использование ФВ ступенчато-прямоугольного профиля позволяет значительно повысить информативность наблюдаемой 
кинетики [13,14]. Этапы решения ОФКЗ численными методами в $[9,10]$ включали: 1) нахождение характеристических скоростей процесса для монотонных участков (стадий) кинетических кривых $[15,16] ; 2)$ интегрирование линейных систем обыкновенных дифференциальных уравнений (ЛСОДУ) [17]; 3) минимизацию ЦФ „рассогласования“ итерационным алгоритмом безградиентной оптимизации Нелдера-Мида $[18,19]$. Эти этапы позволили определить константы скоростей экстралигандирования (аксиального экстракоординирования), отношение начальных концентраций взаимосвязанных центров, а также другие параметры для некоторых металлоорганических люминофоров.

В настоящей работе сообщается об анализе сложных немонотонных кинетических кривых флуоресценции ряда металлопорфиринов в твердых органических полимерных матрицах при ФВ двухступенчатыми прямоугольными импульсами, выполненном на основе решения ОФКЗ. Использованные расчетные методы позволили определить константы скоростей, контролирующие процессы взаимопревращений металлокомплексов тетрапиррольных макроциклов, а также установить механизм фотопревращений, обусловленный обратимым процессом аксиального экстра-лигандирования центрального иона металла. В работе также рассматриваются некоторые важные методические вопросы, связанные с численным решением ОФКЗ.

\section{Экспериментальная часть}

\section{Объекты исследования и техника эксперимента}

Исследовались следующие металлокомплексы порфиринов: $\mathrm{Zn}$-тетра(трет-бутилбензо)порфин $\left(\mathrm{Zn}-(t B u)_{4}-\right.$ ТБП), Zn-тетрабензопорфин (Zn-ТБП), Mg-тетрабензопорфин (Mg-ТБП)и Zn-октаэтилпорфин (Zn-OЭП). Химическая структура $\mathrm{Zn}-(t B u)_{4}$-ТБП показана на вставке рис. 1, а структуры других металлокомплексов представлены в [20]. Электронные спектры поглощения и флуоресценции исследованных соединений приведены в [21]. Окрашенные порфиринами тонкие пленки поливинилбутираля (ПВБ) были приготовлены по методике, описанной в [22], и изучались при комнатной температуре. Металлопорфирины возбуждались в электронноколебательной области полосы Соре лазерным диодом $\left(\lambda_{\text {gen }}=405 \mathrm{~nm}\right)$, генерирующим последовательность импульсов, интенсивность во времени каждого из которых имела прямоугольно двухступенчатый профиль. Длительность импульсов задавалась от $10^{-2} \mathrm{~s}$ и более, а частота их следования - в диапазоне от 1 до $0.01 \mathrm{~Hz}$. Долговременная температурная стабильность профиля интенсивности фотоимпульсов была улучшена схемотехническими приемами термокомпенсации тока лазера. Излучение мощностью $\approx 1 \mathrm{~mW}$ фокусировалось на образце в пятно площадью $\approx 0.1 \mathrm{~mm}^{2}$. Детектирование

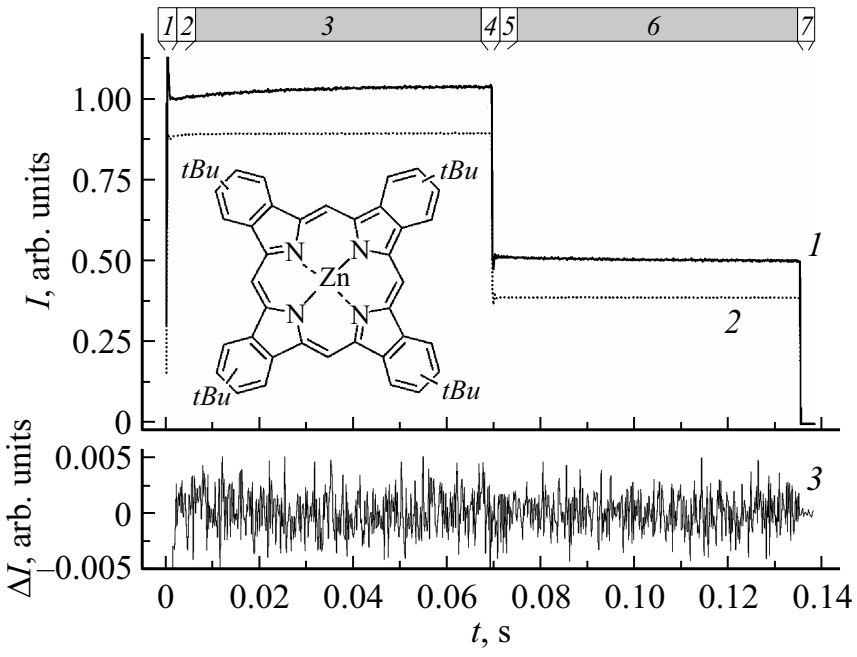

Рис. 1. Кинетика флуоресценции $\mathrm{Zn}-(t B u)_{4}$-ТБП в пленке ПВБ при $T=293 \mathrm{~K}, \lambda_{\text {det }}=632 \mathrm{~nm}$ и $\lambda_{\text {ехс }}=405 \mathrm{~nm}$ (кривая 1$)$, профиль интенсивности ФВ (2), относительная разность интенсивностей экспериментальной и оптимизированной кинетических кривых (3). На вставке изображена структура $\mathrm{Zn}-(t B u)_{4}$-ТБП.

изменения интенсивности флуоресценции металлопорфиринов во времени (далее в тексте, „кинетики флуоресценции“) проводилось в максимуме $0-0$-полосы $S_{1} \rightarrow S_{0^{-}}$ перехода, типичная ширина которой была $350 \mathrm{~cm}^{-1}$, при спектральной ширине щелей регистрирующего монохроматора $($ МДР-23) $\approx 3.0 \mathrm{~nm}$. Одновременно контролировался временной профиль интенсивности лазера путем измерения кинетики люминесценции раствора Родамин Б (Rhodamine B) в этаноле при концентрации менее $10^{-5} \mathrm{~mol} / \mathrm{l}$. Типично, кинетические кривые состояли из $\approx 800$ выборок тока фотоэлектронного умножителя (ФЭУ) с интервалом между ними $\Delta t=10^{-5}-10^{-3} \mathrm{~s}$. Для уменьшения шумов кинетические кривые накапливались и усреднялись типично по 25-100 лазерным импульсам, а профиль интенсивности лазера - по 400-1000 импульсам. Типичная амплитуда шума составляла менее 0.005 максимальной интенсивности кинетических кривых. Более подробные сведения о технике эксперимента приведены в $[10,13,14]$.

\section{Численные расчеты}

Вычислительные процедуры всех этапов ОФКЗ выполнялись в программной среде Scilab версии 5.52 [23]. Характеристические скорости процессов определялись для монотонных участков кинетических кривых сеточными алгоритмами нелинейной регрессии $[15,16]$. Кроме того, аппроксимирующие кривые в ряде расчетов использовались в качестве сглаженных исходных кривых. Интегрирование ЛСОДУ в итерационных циклах оптимизации производилось с помощью Scilab-процедуры LSODE (метод stiff) [17]. Для ускорения интегрирования в явном виде задавался якобиан ЛСОДУ и подбира- 
лись значения вектора ODEOPTIONS, управляющего вычислениями и задаюшего их погрешность. Модельная кинетическая кривая флуоресценция представляла собой функционал в виде суперпозиций решений ЛСОДУ. ЦФ задавалась в виде евклидовой нормы функции „рассогласования“ векторов экспериментальных и модельных кинетик:

$$
Ц \Phi=\sqrt{\sum_{i}\left(I_{i}^{\text {exper }}-I_{i}^{\text {calc }}\right)^{2}}
$$

и минимизировалась алгоритмом безградиентной оптимизации (деформируемого многогранника) Нелдера-Мида [18]. Его выбор был обусловлен возможностью применения к немонотонным и зашумленным функциям, а также простой реализацией и малым количеством итераций, требуемых для достижения критерия завершения расчетов, по сравнению с другими безградиентными алгоритмами [19]. Вместо встроенной в Scilab программы neldermead_new, реализующей алгоритм Нелдера-Мида, была использована процедуpa anms [24], более удобная для модификации. Из-за заметного уменьшения эффективности алгоритма при увеличении размерности задачи количество оцениваемых параметров максимально ограничивалось. Уменьшению их числа способствовало и то, что некоторые параметры могли быть выражены в виде фракции или комбинации других параметров. Типично их количество не превышало 10. В начале оптимизации вектор параметров представлял собой продукт поэлементного произведения вектора их начальных значений на вектор единичных (масштабных), варьируемых алгоритмом множителей. После окончания оптимизации вектор начальных значений параметров поэлементно умножался на вектор оптимизированных множителей. Этот продукт служил вектором начальных параметров для следующей оптимизации. Для вектора, описывающего профиль интенсивности импульса ФВ, оптимизировался свой масштабный множитель. Отметим, что именно использование измеренного профиля ФВ для расчета модельной кинетики флуоресценции позволило аппроксимировать экспериментальную кинетику модельной.

Графический алгоритм решения ОФКЗ методом итерационной оптимизации Нелдера-Мида приведен в [12]. После многократного последовательного запуска процедуры оптимизации или параллельного выполнения нескольких процедур с разными векторами начальных параметров полученные оптимизированные векторы параметров усреднялись. Расчет ОФКЗ для одного соединения требовал не менее 20 запусков процедуры оптимизации или суммарно не менее 50000 итераций. Время одной итерации определялось, главным образом, временем интегрирования ЛСОДУ при порогах абсолютной atol и относительной rtol оцененных ошибок соответственно $10^{-8}$ и $10^{-6}$ и длилось $\sim 1 \mathrm{~s}$ на компьютере с процессором Intel с тактовой частотой $3.5-4.2 \mathrm{GHz}$.
Помимо основных вычислений применялись следующие вспомогательные приемы и методы. Экспериментальные кривые и профиль ФВ заменялись модельными, и производилась оптимизация с целью получения максимального приближения варьируемых параметров к заданным. Это позволило на начальном этапе симуляции убедиться в моноэкспоненциальности всех стадий немонотонной кинетики, а на этапах оценки параметров и выбора физической модели 1) сопоставить результаты оптимизации для разных моделей, 2) выявить степень чувствительности решения к девиации параметров, 3) оценить влияние шума на решение и 4) подобрать параметры алгоритма оптимизации для уменьшения времени расчетов.

На основании обобщения результатов решения ОФКЗ получены следующие достаточно важные сведения. Изменение параметров управления симплексом $\beta$ (expansion, растяжение), $\gamma$ (contraction, сжатие) и $\delta$ (shrink, сужение) в некоторых случаях позволяет уменьшить конечные значения ЦФ. Плодотворной оказалась идея пертурбации центроида (среднеарифметического лучших вертексов) в виде его незначительного искажения в каждом итерационном цикле [25], что позволяет симплексу обходить локальные минимумы. Эффективность метода значительно повышается при уменьшении зашумленности кинетических кривых. Например, при использовании гладких модельных кривых конечные оптимизированные значения параметров практически совпадали со значениями, которые были использованы для синтеза кривых.

Для более простых систем, чем модельные ЛСОДУ, c помощью программы компьютерной алгебры Maxima [26] находились точные аналитические решения, позволившие выявить функциональные связи между скоростями процессов, с одной стороны, константами скоростей и концентрациями молекул взаимопревращающихся центров, с другой.

\section{Результаты и обсуждение}

Для всех образцов наблюдалась повторяемая от импульса к импульсу, зависящая от мощности ФВ кинетика флуоресценции специфического профиля, отличающегося от профиля ФВ. Типичная кинетическая кривая, в частности для $\mathrm{Zn}-(t B u)_{4}$-ТБП, а также профиль интенсивности ФВ приведены на рис. 1.

\section{Определение характеристических скоростей процессов}

На каждой кривой можно было выделить семь стадий, обозначенных цифрами 1-7 (см., как пример, рис. 1). Стадии 1, 4 и 7 соответствовали быстрому изменению интенсивности, как ответ на ступенчатое изменение ФВ.

Стадии 2 и 5 были однозначно охарактеризованы как соответственно фотофизический фейдинг и антифейдинг флуоресценции (ФФ, АФ), которые подробно 


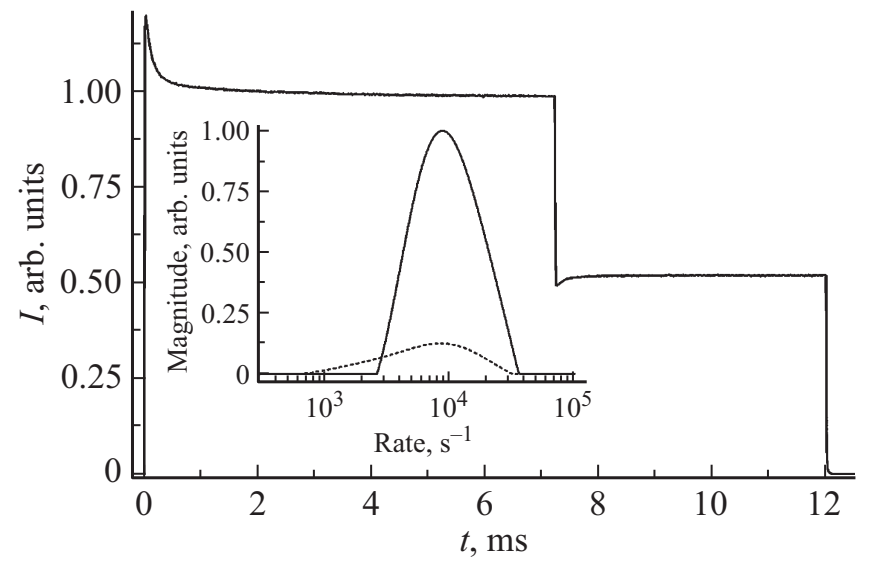

Рис. 2. Кинетика флуоресценции $\mathrm{Zn}-(t B u)_{4}$-ТБП в пленке ПВБ при $T=293 \mathrm{~K}, \lambda_{\operatorname{det}}=632 \mathrm{~nm}$ и $\lambda_{\mathrm{exc}}=405 \mathrm{~nm}$. На вставке изображены результаты CONTIN-расчетов: ФФ (сплошная кривая) и АФ (пунктирная кривая).

рассмотрены в $[13,14]$. ФФ и АФ обусловлены процессами изменения населенности $T_{1}$-состояния с одновременным антибатным изменением населенности основного состояния $S_{0}$, а поэтому и возбужденного $S_{1}$ состояния. Стадии 2 и 5 более наглядно отображаются при укорочении импульса ФВ (рис. 2). Результаты анализа кинетик в виде магнитуд распределения характеристических скоростей ФФ и АФ представлены на вставке рис. 2. Уширение полос обусловлено, главным образом, зашумленностью кривых. Максимумы магнитуд остальных соединений находились в диапазоне скоростей от $6 \cdot 10^{3}$ до $1 \cdot 10^{5} \mathrm{~s}^{-1}$. Из приведенной кинетики можно достаточно точно оценить константы скоростей дезактивации $T_{1}$-состояния $(p)$ и ФВ $\left(k_{\text {ехс }}\right)$. Известно [13], что скорость ФФ $\left(\right.$ rate $\left._{F F}\right)$ определяется выражением rate $_{F F}=p+k_{\mathrm{exc}} Q$, в котором $Q$ - квантовый выход образования $T_{1}$, а относительная амплитуда пика ФФ $\left(\Delta_{F F}\right)$ выражается отношением $\Delta_{F F}=p / k_{\mathrm{exc}} Q$.

Стадии 3 и 6 демонстрировали соответственно относительно медленное увеличение и уменьшение флуоресценции и свидетельствовали о том, что наряду с быстрыми фотофизическими процессами стадий 2 и 5 в системе протекают медленные фотопроцессы иной природы. Типичные скорости стадии 3 составляли $\sim 35-50 \mathrm{~s}^{-1}$, а стадии $6-\sim 13-25 \mathrm{~s}^{-1}$. Значительная схожесть кинетики флуоресценции на этих стадиях и кинетики люминесценции комплекса $\mathrm{Eu}^{3+}[10]$ позволила предположить, что ансамбль флуорофоров химически неоднороден и некоторые его подансамбли превращаются друг в друга. Дополнительным аргументом в пользу этой гипотезы свидетельствует кинетика NHфототаутомерии свободных оснований порфиринов при низких температурах [27]. Кроме того, численное и точное аналитическое решение для населенности $S_{1}$-уровня (интенсивности флуоресценции) при ступенчатом ФВ в приближении трехуровневой модели, т.е. для однородного ансамбля, имеет вид спадающей монотонной кривой $[9,28]$. Добавление дополнительных уровней в такую модель изменяет только характер монотонности.

\section{Оценка параметров моделей и констант скоростей}

Для выяснения причин немонотонности кинетики, т.е. появления стадий 3 и 6, был применен формальнокинетический подход. Рассматривалось несколько гипотетических моделей фотопроцессов, состоящих, исходя из соображений минимализма, из двух типов химически неэквивалентных комплексов (К1 и К2). Отличие моделей состояло в реализации различных путей дезактивации энергии ФВ. Схема энергетических уровней и путей дезактивации энергии ФВ одной из них, описывающей превращения К $1 \leftrightarrow$ К 2 с участием переходов $T_{1}^{(1)} \leftrightarrow T_{1}^{(2)}$ и $S_{0}^{(1)} \leftrightarrow S_{0}^{(2)}$ (модель М-1), приведена на рис. 3 .

Предполагалось, что уровни $S_{1}^{(1)}$ и $S_{1}^{(2)}$ заселяются соответственно со скоростями ФВ $k_{\mathrm{exc} 1}$ и $k_{\mathrm{exc} 2}$ напрямую, а не в результате заселения высоколежащих уровней $S_{i}^{(1)}$ и $S_{i}^{(2)}$ (на схеме не показаны) с последующей быстрой внутренней безызлучательной конверсией $\left.S_{i}^{(1)} \sim\right\rangle S_{1}^{(1)}$ и $\left.S_{i}^{(2)} \sim\right\rangle S_{1}^{(2)}$. Такое упрощение уменьшало количество оцениваемых параметров без понижения точности решения ОФКЗ. Математическая модель в виде задачи Коши, не учитывающая вынужденное испускание, межмолекулярные взаимодействия и необратимые фотопроцессы,

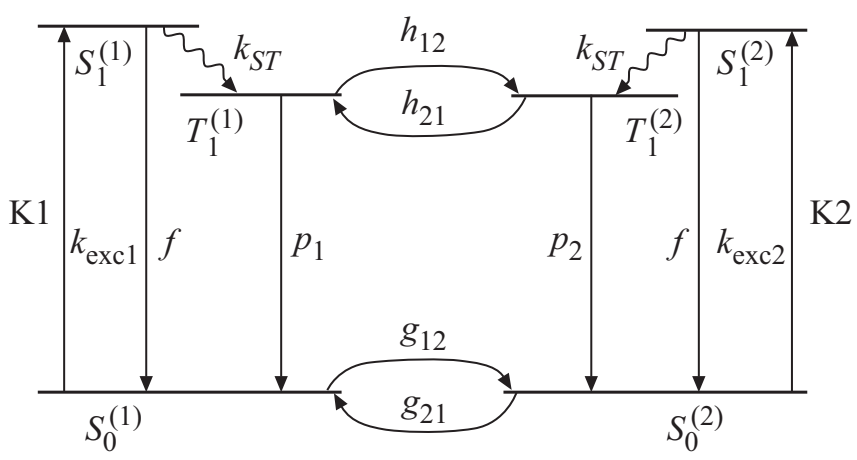

Рис. 3. Схема энергетических уровней и переходов между ними для комплексов К1 и К2 металлопорфиринов при реализации обратимых фотопревращений К1 M-1. Прямыми и кривыми со стрелками показаны константы скорости: $k_{\operatorname{exc} 1}$ и $k_{\operatorname{exc} 2}-\Phi \mathrm{B}, f-$ суммарной дезактивации $S_{1}$ состояния, $k_{S T}$ - интеркомбинационной конверсии $S_{1} \sim T_{1}, p_{1}$ и $p_{2}$ - суммарной дезактивации $T_{1}$-состояния, $h_{12}$ и $h_{21}-$ обратимых переходов $T_{1}^{(1)} \leftrightarrow T_{1}^{(2)}, g_{12}$ и $g_{21}-$ обратимых темновых переходов $S_{0}^{(1)} \leftrightarrow S_{0}^{(2)}$. 
Статистически усредненные значения оцененных параметров и констант скоростей моделей М-1-M-4*

\begin{tabular}{l|c|c|c|c|c|c|c|c|c|c}
\hline $\begin{array}{c}\text { Соединение } \\
(\text { модель })\end{array}$ & $n_{1} / n_{2}$ & $r_{1} / r_{2}$ & $k_{\mathrm{exc1},} \mathrm{s}^{-1}$ & $k_{\mathrm{exc2}, \mathrm{s}^{-1}}$ & $p_{1}, \mathrm{~s}^{-1}$ & $p_{2}, \mathrm{~s}^{-1}$ & $h_{12}, \mathrm{~s}^{-1}$ & $h_{21}, \mathrm{~s}^{-1}$ & $g_{12}, \mathrm{~s}^{-1}$ & $g_{21}, \mathrm{~s}^{-1}$ \\
\hline $\mathrm{Zn}-(t B u)_{4}$-ТБП (M-1) & 1.2 & 0.20 & 900 & 1500 & 11000 & 4500 & 165 & 55 & 11 & 13 \\
\hline $\mathrm{Zn}-(t B u)_{4}$-ТБП (M-2) & 2.0 & 0.24 & 1400 & 1600 & 13000 & 4000 & 100 & 65 & 7 & 13 \\
\hline $\mathrm{Zn-}(t B u)_{4}$-ТБП (M-3) & 7.5 & 0.23 & 2150 & 900 & 9350 & 1850 & $5.3 \cdot 10^{5}$ & $1.4 \cdot 10^{5}$ & 7 & 50 \\
\hline $\mathrm{Zn}-(t B u)_{4}$-ТБП (M-4) & 11 & 0.16 & 2350 & 750 & 9600 & 1480 & $3.8 \cdot 10^{5}$ & $2.1 \cdot 10^{5}$ & 5 & 55 \\
\hline $\mathrm{Zn-ТБП} \mathrm{(М-1)}$ & 1.2 & 0.15 & 1000 & 1500 & 12000 & 4700 & 170 & 50 & 10 & 12 \\
\hline Мg-ТБП (М-1) & 1.4 & 0.4 & 1300 & 2100 & 10700 & 4700 & 250 & 30 & 8 & 5 \\
\hline Zn-ОЭП (М-1) & 1.0 & 0.15 & 1050 & 1500 & $6 \cdot 10^{4}$ & $2 \cdot 10^{4}$ & 135 & 30 & 6 & 7
\end{tabular}

Примечание. *Различие моделей приведено после описания ЛСОДУ (1).

имела вид ЛСОДУ:

$$
\left\{\begin{array}{l}
\frac{d\left[S_{0}^{(1)}\right]}{d t}=-\left(k_{\operatorname{exc} 1}+g_{12}\right)\left[S_{0}^{(1)}\right]+f\left[S_{1}^{(1)}\right]+p_{1}\left[T_{1}^{(1)}\right]+g_{21}\left[S_{0}^{(2)}\right], \\
\frac{d\left[S_{1}^{(1)}\right]}{d t}=k_{\operatorname{exc} 1}\left[S_{0}^{(1)}\right]-\left(f+k_{S T}\right)\left[S_{1}^{(1)}\right] \\
\frac{d\left[T_{1}^{(1)}\right]}{d t}=k_{S T}\left[S_{1}^{(1)}\right]-\left(p_{1}+h_{12}\right)\left[T_{1}^{(1)}\right]+h_{21}\left[T_{1}^{(2)}\right] \\
\frac{d\left[S_{0}^{(2)}\right]}{d t}=-\left(k_{\operatorname{exc} 2}+g_{21}\right)\left[S_{0}^{(2)}\right]+f\left[S_{1}^{(2)}\right]+p_{2}\left[T_{1}^{(2)}\right]+g_{12}\left[S_{0}^{(1)}\right] \\
\frac{d\left[S_{1}^{(2)}\right]}{d t}=k_{\operatorname{exc} 2}\left[S_{0}^{(2)}\right]-\left(f+k_{S T}\right)\left[S_{1}^{(2)}\right] \\
\frac{d\left[T_{1}^{(2)}\right]}{d t}=k_{S T}\left[S_{1}^{(2)}\right]-\left(p_{2}+h_{21}\right)\left[T_{1}^{(2)}\right]+h_{12}\left[T_{1}^{(1)}\right]
\end{array}\right.
$$

со следующими начальными условиями и замкнутостью:

$$
\begin{aligned}
& {\left[S_{0}^{(1)}(0)\right]+\left[S_{0}^{(2)}(0)\right]=n_{1}+n_{2}=\left[S_{0}^{(1)}(t)\right]+\left[S_{1}^{(1)}(t)\right]+} \\
& +\left[T_{1}^{(1)}(t)\right]+\left[S_{0}^{(2)}(t)\right]+\left[S_{1}^{(2)}(t)\right]+\left[T_{1}^{(2)}(t)\right]=\mathrm{const} .
\end{aligned}
$$

Параметры $n_{1}$ и $n_{2}$ - исходные концентрации К1 и К2 соответственно. В других моделях $h_{12}$ и $h_{21}$ учитывали принципиально другие переходы: $T_{1}^{(1)} \rightarrow S_{0}^{(2)}, T_{1}^{(2)} \rightarrow S_{0}^{(1)}$ $(\mathrm{M}-2) ; S_{1}^{(1)} \leftrightarrow S_{1}^{(2)}(\mathrm{M}-3) ; S_{1}^{(1)} \rightarrow S_{0}^{(2)}, S_{1}^{(2)} \rightarrow S_{0}^{(1)}(\mathrm{M}-4)$.

По причине того, что кинетические профили измерялись с временным разрешением, не позволяющим оценить скорости процессов стадий 1,4 и 7, константы которых, как следует из теоретического анализа [25], превышают сумму констант $f$ и $k_{S T}$, последние задавались одинаковыми для К1 и К2. Значения $f=2 \cdot 10^{7}$ и $k_{S T}=3 \cdot 10^{8} \mathrm{~s}^{-1}$ и соответственно квантовый выход образования триплетных состояний $Q=k_{S T} /\left(k_{S T}+f\right) \approx 0.9$ [21] задавались одинаковыми для всех соединений. Такое упрощение, с одной стороны, уменьшило число оцениваемых параметров, а с другой, минимально повлияло на результаты. Начальные значения констант скоростей $p_{1}$ и $p_{2}$ задавались и контролировались, чтобы не превышать величину rate $_{F F}$.

При отсутствии $Ф В$ населенности $S_{0}$-уровней К1 и К2 - равновесные и определяются равенством скоростей их взаимопревращений: $g_{12}\left[S_{0}^{(1)}(t)\right]=g_{21}\left[S_{0}^{(2)}(t)\right][29]$.
Это равенство позволило дополнительно уменьшить число оцениваемых параметров на единицу.

Интенсивность флуоресценции задавалась в виде суперпозиции решений ЛСОДУ $(1): \quad I(t)=r_{1} \times$ $\times\left[S_{1}^{(1)}(t)\right]+r_{2}\left[S_{1}^{(2)}(t)\right]$. Параметры $r_{1}$ и $r_{2}$ - относительные (излучательные) вклады в $f$ соответственно К1 и К2. Параметры $n_{1}, n_{2}$ и $r_{1}$ оценивались при оптимизации, а $r_{2}$ полагался равным 1 в силу достаточности задания только отношения $r_{1} / r_{2}$.

Для установления зависимости скорости протекания процессов на стадиях 3 и 6 от параметров моделей был проведен теоретический анализ аналитического решения упрощенной 4-уровневой модели ЛСОДУ, близкой к ЛСОДУ (1), поскольку последняя не имеет явного аналитического решения. Упрощение сводилось к парному объединению второго-третьего и пятого-шестого уравнений системы (1), заданию равенств $k_{\mathrm{exc1}}=k_{\mathrm{exc2}}=k_{\mathrm{exc}}$ и $p_{1}=p_{2}=p$, а также удалению $f$ и $k_{S T}$. После упрощения система была подобна приведенной в [10] системе (1). В результате приведения громоздких решений и пренебрежения заведомо малыми членами (при разложении в ряд некоторых выражений) оказалось, что скорости процессов в рамках исходной модели М-1 при $k_{\mathrm{exc} 1} \approx k_{\mathrm{exc} 2}$ и $p_{1} \approx p_{2}$ с относительной погрешностью не более 0.1 определяются выражением

$$
\operatorname{rate}_{3,6}^{\mathrm{M}-1} \approx h_{12}+h_{21}-\frac{h_{12}+h_{21}-\left(g_{12}+g_{21}\right)}{1+\Delta} .
$$

В (2) $\Delta=k_{\text {exc }} Q / p$, а $k_{\text {ехс }}$ и $p$ могут быть заменены среднегеометрическими значениями соответственно $k_{\text {exc1 }}$, $k_{\text {exс2 }}$ и $p_{1}, p_{2}$ в случае парной близости их значений. Для сохранения приближенного равенства в (2) $\Delta$ не должно быть намного больше 1. Если триплетные уровни не заселены, т.е. $k_{\text {exc }} Q \ll p$, то rate $3,6 \approx g_{12}+g_{21}$. Если $k_{\mathrm{exc}} Q \gg p$, то rate $_{3,6} \approx h_{12}+h_{21}$. Параметр $\Delta$ близок к $\Delta_{F F}$, а как показано в [13], $\Delta_{F F}$ определяется отношением интенсивности пика стадии 2 к её минимуму и показывает относительную населенность $T_{1}$-уровня. В наших исследованиях $\Delta$ не превышала 0.3 . 
В случае М-3 выражение для скоростей процессов на стадиях 3 и 6 было получено из такой же упрощенной модели и имело схожий с (2) вид:

$$
\operatorname{rate}_{3,4}^{\mathrm{M}-3} \approx h_{12}+h_{21}-\frac{h_{12}+h_{21}-\left(g_{12}+g_{21}\right)}{1+\frac{k_{\text {exc }}}{f}} .
$$

Экспериментальные интенсивности ФВ были таковы, что всегда выполнялось $k_{\text {exс }} \ll f$, вследствие чего в случае M-3 относительно малая скорость rate 3,6 могла быть результатом разности большого значения суммы $h_{12}+h_{21}$ и величины второго слагаемого, очень близкой к этой сумме. Выражения, подобные (2) и (3), для M-2 и M-4 не были получены по причине отсутствия аналитического решения для их упрощенных моделей. Однако численное моделирование показало, что они весьма близки соответственно к (2) и (3).

Результаты определения констант скоростей внутрикомплексных процессов, взаимопревращений, а также отношения $n_{1} / n_{2}$ и $r_{1} / r_{2}$ для исследованных соединений приведены в таблице. Параметры и константы скоростей фотопроцессов всех четырех моделей представлены только для $\mathrm{Zn}-(t B u)_{4}$-ТБП. Относительная погрешность табличных данных \pm 0.25 . Выражения (2) и (3) при подстановке табличных значений дают величины, согласующиеся со скоростями процессов, которые были получены из экспериментальных кривых.

Квантовая эффективность процессов превращений К в $\mathrm{K}_{j}(i, j=1,2$ и $i \neq j)$ для $\mathrm{M}-1, \mathrm{M}-2$ и $\mathrm{M}-3, \mathrm{M}-4$ может быть представлена соответственно следующими выражениями:

$$
L_{i j}=Q \frac{h_{i j}}{p_{i}+h_{i j}}, \quad L_{i j}=\frac{h_{i j}}{f+k_{i s c}+h_{i j}} .
$$

Очевидно, что для всех моделей $L_{i j} \approx 0.01$ (1 акт превращения на 100 актов дезактивации $S_{1^{-}}$или $T_{1}$ состояния).

\section{Дискриминация моделей}

Все модели показали практически одинаковую согласованность профилей экспериментальных и оптимизированных кинетических кривых на стадиях 3-6, о чем свидетельствовала относительная разность сопоставляемых значений, которая не превышала \pm 0.005 (рис. 1, кривая 3) и близкие значения ЦФ, отличавшиеся по величине не более, чем на 0.01. Поэтому принять решение в пользу той или иной модели по критерию согласованности профилей кривых и малости ЦФ не представлялось возможным. Выполненные с целью выяснения принципиальной возможности дискриминации по значениям ЦФ расчеты для М-1 - M4 с модельными гладкими (незашумленными) кинетическими кривыми показали, что различить кинетики можно только при условии полного отсутствия шумов анализируемых кривых, а также очень высокой точности (достаточного числа значащих цифр) сопоставляемых (анализируемых и расчетных) кривых. Результаты симуляции явно свидетельствуют о математическом подобии решений для всех моделей. Уменьшение шумов экспериментальных кривых до экстремально низких (нулевых) значений и их измерение с очень высокой точностью в наших экспериментах практически не представлялось возможным. По этой причине дискриминация производилась исходя из физического смысла значений оцененных констант скоростей и параметров.

Согласно расчетам, для М-1 характерна близость по порядку величины значений констант скоростей $h_{12}$ и $h_{21}$, а также $g_{12}$ и $g_{21}$. Очевидно, что такая ситуация реализуется при практическом резонансе энергий триплетных $T_{1}^{(1)}$ - и $T_{1}^{(2)}$ - и синглетных $S_{0}^{(1)}$ - и $S_{0}^{(2)}$ уровней. Из анализа табличных значений следует, что скорость безызлучательной дезактивации К1 выше, чем К2, как в системе синглетных $\left(r_{1}<r_{2}\right)$, так и в системе триплетных $\left(p_{1}>p_{2}\right)$ уровней. В работе [30] на примере молекул Zn-OЭП экспериментально показано, что процесс экстралигандирования центрального иона металла приводит к росту вероятности безызлучательной дезактивации $T_{1}$-состояния, что обусловлено участием экстралигандов в размене энергии электронного возбуждения и усилением спин-орбитального взаимодействия в результате нарушения плоскостности макроцикла. На этом основании можно сделать однозначный вывод, что в М-1 К1 является монолигандированной формой, а К2 - нелигандированной. Соответственно константы скоростей $g_{12}$ и $h_{12}$ характеризуют процесс отсоединения экстра-лиганда, а $g_{21}$ и $h_{21}$ - присоединения. Вероятнее всего, в качестве экстралигандов могли быть вода, этанол и другие мобильные неконтролируемые примеси, присутствующие в ПВБ и обладающие координационной способностью [31]. Дополнительным подтверждением экстралигандирования является схожесть наблюдаемых немонотонных кинетик флуоресценции металлопорфиринов с кинетикой люминесценции европиевого комплекса $[10]$, где сделано заключение, что немонотонность кривой отражает обратимые процессы в редкоземельном комплексе, связанные с изменением его лигандного состава. Исходные равновесные концентрации К1 и К2 практически одинаковы $\left(n_{1} / n_{2} \approx 1.2\right)$. Увеличение интенсивности флуоресценции на стадии 3 обусловлено увеличением концентрации (вследствие того, что $h_{12}>h_{21}$ ) более эффективно флуоресцирующего K2 $\left(r_{2}>r_{1}\right)$.

Как видно из таблицы, М-2 показала близкие к М-1 значения оцененных констант скоростей и параметров. Это является следствием того, что в этих моделях фотопревращения К1↔К2 происходят с участием долгоживущих $T_{1}$-состояний, и в обоих случаях имеют место процессы экстралигандирования со схожими механизмами. Из таблицы видно, что для М-3 и М-4 значения $h_{12}$ и $h_{21}$ на два-три порядка меньше каждой из констант $f$ и $k_{S T}$ и на один-два порядка превосходят $p_{1}$ и $p_{2}$. При ФВ лиганды в $S_{1}$-состояниях отсоединяются 
быстрее $\left(h_{12}>h_{21}\right)$, чем присоединяются. Наоборот, в отсутствие ФВ гораздо более стабильным является экстралигандированный К1 ( $n_{1}$ на порядок превышает $\left.n_{2}\right)$.

Несмотря на то, что модели М-3 и M-4 показали высокую согласованность профилей экспериментальных и оптимизированных кинетических кривых на стадиях 3-6, их реальное существование представляется маловероятным. Во-первых, исключительно высокие скорости процессов отсоединенияприсоединения экстралигандов к металлопорфирину в $S_{1}$-состоянии в твердой полимерной матрице не могут реализовываться в условиях затрудненного диффузного движения как примесей (экстралигандов), так и внедренных порфиринов. Во-вторых, трудно объяснимой представляется значительная разница между $h_{12}, \quad h_{21}$ и $g_{12}, g_{21}$. В-третьих, неравенство $g_{12}<g_{21}$ и, как следствие, преобладание населенности К1 в $S_{0}$-состоянии $\left(n_{1}>n_{2}\right)$ должно, согласно [30], вызвать заметный батохромный сдвиг длинноволновой 0-0-полосы поглощения этого экстралигандированного комплекса (для экстралигандированного $\mathrm{Zn}-\mathrm{O} Э$ подобный батохромный сдвиг достигал $\sim 6-10 \mathrm{~nm}$ при $293 \mathrm{~K}$ при ширине 0-0-полос флуоресценции $\left.\sim 330-350 \mathrm{~cm}^{-1}[30]\right)$. В итоге суперпозиция батохромно смещенной полосы поглощения К1 и полосы К2 должна привести к заметному уширению суммарной длинноволновой $0-0$-полосы поглощения и 0-0-полосы флуоресценции, что экспериментально не наблюдалось. Расчет показал, что нелигандированный комплекс К2 обладает более эффективной флуоресценцией, чем лигандированный К1, что согласуется с данными [30].

Обнаруженная „жесткая“ зависимость скорости процессов на стадиях 3 и 6 от параметров, выраженная (3), также указывает не в пользу М-3 и М-4.

\section{Выводы}

Анализ сложных немонотонных кинетических кривых флуоресценции ряда металлопорфиринов в твердых органических полимерных матрицах выполнен на основе полного решения ОФКЗ. Оценка констант скоростей и параметров многопараметрической ОФКЗ для исследованных систем, сформулированных в виде ЛСОДУ для четырех моделей фотопроцессов, произведена с использованием безградиентного алгоритма НелдераМида, показавшего высокую эффективность и надежность. При оценке решений учитывалась малость значения ЦФ, детальная согласованность профилей экспериментальных и оптимизированных кинетических кривых, а также близость значений оцененных констант скоростей и параметров к экспериментальным данным при сохранении физического смысла гипотетической модели.

Использованные методы ОФКЗ позволили определить параметры и константы скоростей процессов, контро- лирующих взаимопревращения металлокомплексов тетрапиррольных макроциклов. Сделан вывод о том, что взаимопревращения обусловлены обратимым процессом аксиального экстралигандирования центрального иона металла порфирина, т.е. существованием двух типов химически неэквивалентных комплексов (К1 и К2). Дискриминация моделей позволила заключить, что обратимый процесс присоединения-отсоединения экстралиганда металлопорфирином происходит с участием $T_{1}$-состояния (модели М-1 и М-2) и протекает в среднем на порядок быстрее, чем в $S_{0}$-состоянии. Процессы аксиального экстракоординирования значительно как минимум на два порядка более медленные, чем внутримолекулярные процессы дезактивации энергии ФВ. Результаты представленных исследований позволили доказательно обосновать протекание фотопроцессов в конденсированных средах по общепризнанным кинетическим схемам.

Выполненные исследования также имеют методологическое значение. Реализация ОФКЗ позволяет судить о характере взаимодействия примесей или локального матричного окружения с внедренными флуорофорами и тем самым обосновывать временную эволюцию физико-химических свойств и качества активированных оптически-прозрачных материалов.

\section{Конфликт интересов}

Авторы заявляют, что у них нет конфликта интересов.

\section{Список литературы}

[1] Димитров В.И. Простая кинетика. Сибирское отделение. Новосибирск: Наука, 1982. 382 с.

[2] Денисов А.М. Введение в теорию обратных задач. Учебное пособие. М: Изд-во МГУ, 1994. 208 р.

[3] Спивак С.И, Губайдулин И.М., Вайман Е.В. Обратные задачи химической кинетики. Учебное пособие. Уфа: РИОБашГУ, 2003. 110 c.

[4] Kabanikhin S.I. Inverse and Ill-posed Problems: Theory and Applications. Series 55. Berlin, Boston: de Gruyter $\mathrm{GmbH}$. 2012. 459 p. ISBN 978-3-11-022400-9. doi 10.1515/9783110557350

[5] Milstein J. // Modelling of Chemical Reaction Systems. Chem. Phys. Series 18. Heidelberg: Springer-Verlag, 1981. P. 92. doi 10.1007/978-3-642-68220-9

[6] Carlson G.M., Provder T. // Computer Applications in the Polymer Laboratory. ACS Symposium Series. 1986. Ch. 21. V. 313. P. 241. doi $10.1021 /$ bk-1986-0313.ch021

[7] Pomerantzev A.L, Rodionova O.E. // Polymer and Biopolymer Analysis and Characterization. 2007. Ch. 13. P. 179. ISBN: 978-1-60021-608-4

[8] Kabanikhin S., Krivorotko O., Mortier A. et al. // Сибирский научный медицинский журнал. 2016. Т. 6. № 1. С. 29.

[9] Станишевский И.В., Арабей С.М., Павич Т.А. // Материалы XI Междунар. науч.-техн. конфер. „Квантовая электроника“. Минск: РИВШ, 2017. С. 39. ISBN 978-985585-091-5 
[10] Станишевский И.В., Павич Т.А., Арабей С.М. // Опт. и спектр. 2019. Т. 126. № 2. С. 133.

doi 10.21883/OS.2019.02.47194.176-18;

Stanishevsky I.V, Pavich T.A, Arabei S.M. // Opt. Spectrosc. 2019. V. 126. N 2. P. 111. doi 10.1134/S0030400X1902022X

[11] Станишевский И.В., Арабей С.М. // Материалы ХІІ Междунар. науч.-техн. конфер. „Квантовая электроника“. Минск: РИВШ, 2019. С. 64.

[12] Станишевский И.В. // Материалы XII Междунар. науч.техн. конфер. „Квантовая электроника“. Минск: РИВШ, 2019. C. 80.

[13] Станишевский И.В., Соловьев К.Н., Арабей С.М., Чернявский В.A. // Журнал приклад. спектроск. 2013. Т. 80. № 3. C. 368; Stanishevsky I.V., Solovyov K.N., Arabei S.M., Chernyavsky V.A. // J. Appl. Spectrosc. 2013. V. 80. N 3. P. 357. doi 10.1007/s10812-013-9773-9

[14] Станишевский И.В., Арабей С.М., Чернявский В.А., Соловьев К.Н. // Опт. и спектр. 2016. Т. 121. № 5. C. 770; Stanishevsky I.V., Arabei S.M., Chernyavsky V.A., Solovyov K.N. // Opt. Spectrosc. 2016. V. 121. N 5. P. 722. doi 10.1134/S0030400X16110199

[15] Provencher S.W. // Comp. Phys. Commun. 1982. V. 27. N 3. P. 213. doi 10.1016/0010-4655(82)90173-4

[16] Elster C., Honerkamp J., Weese J. // Rheologica Acta. 1992. V. 31. N 2. P. 161 . doi $10.1007 / \mathrm{BF} 00373238$

[17] Hindmarsh A.C. // Scientific Computing. Amsterdam: NorthHolland, 1983. P. 55.

[18] Nelder J.A., Mead R. // Comput. J. 1965. V. 7. P. 308. doi 10.1093/comjn1/7.4.308

[19] Колесникова Е.В., Колесникова Л.И., Русин Л.Ю. // Физико-химическая кинетика в газовой динамике. 2010. T. 10. C. 495.

[20] Койфман О.И., Семейкин А.С., Березин Б.Д. // Порфирины: структура, свойства, синтез. М.: Наука, 1985. С. 205.

[21] Кузьмицкий В.А., Соловьев К.Н., Цвирко М.П. // Порфирины: спектроскопия, электрохимия, применение. М.: Наука, 1987. C. 7.

[22] Павич Т.А., Арабей С.М., Соловьев К.Н. // Журнал приклад. спектроск. 2018. Т. 85. № 1. С. 5; Pavich T.A., Arabei S.M., Solovyov K.N. // J. Appl. Spectrosc. 2018. V. 85. N 1. P. 1. doi 10.1007/s10812-018-0603-y

[23] Scilab. [Электронный ресурс] Режим доступа: https://en.wikipedia.org/wiki/Scilab.

[24] Gao F., Han L. // Comput. Optim. Appl. 2012. V. 51. N 1. P. 259. doi 10.1007/s10589-010-9329-3

[25] Fajfar I., Bürmen A., Puhan J. // Optimization Letters. 2019. V. 13. N 5. P. 1011. doi 10.1007/s11590-018-1306-2

[26] Maxima. [Электронный ресурс] Режим доступа: https://en.m.wikipedia.org/wiki/Maxima_(software).

[27] Arabei S.M., Galaup J.-P., Solovyov K.N., Donyagina V.F. // Chem. Phys. 2005. V. 311. N 3. P. 307. doi 10.1016/j.chemphys.2004.10.045

[28] Станишевский И.В. // Материалы XI Междунар. науч.техн. конфер. „Квантовая электроника“. Минск: РИВШ, 2017. C. 369. ISBN 978-985-585-091-5

[29] Станишевский И.В., Соловьев К.Н. // Опт. и спектр. 2004. T. 96. № 2. C. 255; Stanishevsky I.V., Solovyov K.N. // Opt. Spectrosc. 2004. V. 96. N 2. P. 221. doi 10.1134/1.1651247
[30] Кнюкшто В.Н, Сагун И.Е, Шульга А.М., Зенькевич Э.И. // Журнал приклад. спектроск. 1998. Т. 65. № 6. С. 900; Knyukshto V.N., Shul'ga A.M., Sagun E.I., Zen'kevich E.I. // J. Appl. Spectrosc. 1998. V. 65. N 6. P. 943. doi 10.1007/BF02675753

[31] Mamardashvili N.Zh., Borovkov V.V., Mamardashvili G.M., Inoue Y., Koifman O.I. // Chemical Processes with Participation of Biological and Related Compounds. Biophysical and Chemical Aspects of Porphyrins, Pigments, Drugs, Biodegradable Polymers, and Nanofibers. Leiden: Koninklijke Brill, 2008. P. 117. 